\title{
Shexiang Tongxin dropping pill protects against isoproterenol- induced myocardial ischemia in vivo and in vitro
}

\author{
Jianyong $\mathbf{Q i}^{1,2, *}$, Wenjun Pan ${ }^{1,2, *}$, Yafang Tan ${ }^{1,2, *}$, Jiaru Luo ${ }^{1,2, *}$, Dancai Fan ${ }^{1,2}$, Juan $\mathbf{Y u}^{3}$, \\ Jiashin $\mathbf{W u}^{4}$ and Minzhou Zhang ${ }^{1,2}$ \\ ${ }^{1}$ AMI Key Laboratory of Chinese Medicine in Guangzhou, The Second Affiliated Hospital of Guangzhou University of Chinese \\ Medicine, Guangdong Province Hospital of Chinese Medicine, Guangzhou 510006, China \\ ${ }^{2}$ Intensive Care Research Team of Traditional Chinese Medicine, The Second Affiliated Hospital of Guangzhou University of \\ Chinese Medicine, Guangdong Province Hospital of Chinese Medicine, Guangzhou 510006, China \\ ${ }^{3}$ Animal Center, The Second Affiliated Hospital of Guangzhou University of Chinese Medicine, Guangdong Province Hospital \\ of Chinese Medicine, Guangzhou 510006, China \\ ${ }^{4}$ Department of Pharmaceutical Sciences, College of Pharmacy, Northeast Ohio Medical University, Rootstown, Ohio 44272, \\ USA \\ * These authors have contributed equally to this work and share the first authorship \\ Correspondence to: Minzhou Zhang, email: minzhouzhang8@163.com \\ Keywords: myocardial ischemia; isoproterenol; signaling pathway; rat; Shexiang Tongxin dropping pill \\ Received: July 27, $2017 \quad$ Accepted: October 28, $2017 \quad$ Published: November 14, 2017 \\ Copyright: Qi et al. This is an open-access article distributed under the terms of the Creative Commons Attribution License 3.0 (CC \\ BY 3.0), which permits unrestricted use, distribution, and reproduction in any medium, provided the original author and source are \\ credited.
}

\section{ABSTRACT}

Shexiang Tongxin dropping pill (STDP) is a formulae of Chinese Medicine commonly used to treating angina pectoris in China. However, its mechanism of action is still yet unclear. This study investigated the roles of STDP on myocardial ischemia injury. We constructed a rat model of myocardial injury (isoproterenol subcutaneous injection, i.h, $85 \mathrm{mg} / \mathrm{kg} / \mathrm{day}$ for 2 days), and compared among 4 groups: CON (control), ISO (ischemic injury model), MET (metoprolol), and STDP. Serum contents of Troponin I (CTnI), creatine kinase (CK), CK-MB, lactate dehydrogenase (LDH), alpha-hydroxybutyric dehydrogenase (a-HBD), and Aspartate Aminotransferase were detected and five STDP doses (1, 10, 100, 1000 and 10000 $\mathrm{mg} / \mathrm{kg} / \mathrm{day}$ ) were chosen to obtain a dose-response curve. Western-blot was used to detect phosphorylations of extracellular signal-regulated kinase 1/2 (ERK1/2), protein kinase B (AKT), and camodulin kinase II (CamkII). Furthermore, an ERK1/2 inhibitor PD98059, a phosphatidylinositol-3-kinase inhibitor, LY294002, and a CamKII inhibitor, KN-93 were administered i.h. Results: cTnI, CK, CK-MB, a-HBD, and LDH were significantly lower in STDP than ISO $(P<0.05)$. STDP exhibited a dose-dependent effect with a half maximal inhibitory concentration of $42 \mathrm{mg} / \mathrm{kg} / \mathrm{day}$. Phosphorylation of ERK1/ 2 was enhanced in the STDP group (vs. ISO, P<0.05), while AKT and CamkII were not changed. Further, the protective effects of STDP were offset by PD98059 administration i.h. In conclusion, STDP protected against the ISO-induced myocardial ischemic injury via an ERK1/2 signaling pathway, which provided a mechanism to support clinical applications of STDP as treatment for ischemic heart disease.

\section{INTRODUCTION}

Myocardial ischemia and infarction are the leading causes of morbidity and mortality in U.S. [1] and are also becoming major causes of death worldwide due to prevalence of obesity, high blood pressure, and unhealthy lifestyles [2]. Although percutaneous coronary intervention (PCI) is effective in reducing the consequences of myocardial ischemia and infarction, PCI procedures also frequently cause reperfusion injury. Moreover, sudden 
cardiac death can occur before patients have the chance of receiving PCI operation. Therefore, drug treatment is still a convenient and important method to rescue patients from acute myocardial ischemia and infarction. Despite advances in basic research and clinical improvements, current drug therapies are still insufficient to manage and treat the disease.

ShexiangTongxin dropping pill (STDP), an herb formula of Traditional Chinese Medicine, has been widely used for treating angina pectoris in Chinese clinics and hospitals during the last 2 decades. It was reported that STDP could effectively alleviate unstable angina pectoris and reduce major adverse cardiovascular events in patients when combined with conventional therapies [3]. It was also demonstrated that STDP could enhance cardiac function in patients with heart failure [4].

Although STDP is a complex herb formula, ultraperformance liquid chromatography coupled with triple-quadrupole tandem mass spectrometry (UPLCQqQ-MS/MS) Quantitative Analysis revealed 13 major chemical constituents in STDP: ginsenoside Rg1, ginsenoside $\mathrm{Rg} 3$, cinobufagin, arenobufagin, bufalin, resibufogenin, tanshinone IIA, taurine, astragaloside, tauroursodeoxycholic acid, taurocholic acid, cholic acid, deoxycholic acid, and chenodeoxycholic acid. (Figure 1) [5]. Artificial musk administration was reported to protect against myocardial ischemic injury in animal studies [6]. Fel Ursi showed various pharmacological activities, such as antimicrobial, anti-inflammatory, treating hypertension, liver fibrosis, dyslipidemia, thrombosis, and tumor growth, etc $[7,8]$.

Despite broad applications of STDP in treating angina pectoris in Chinese clinics and hospitals, seldom is reported about the underlying mechanisms of STDP treating myocardial ischemic injury. The present study investigated mechanisms of STDP's cardio-protective effects using an ISO-induced myocardial ischemic injury model in rats.

\section{RESULTS}

\section{STDP significantly reduced the ISO-induced myocardial ischemia in vivo}

To explore the effects of STDP on myocardial injury in vivo, we created a rat ischemia model by ISO subcutaneous injection (i.h, $85 \mathrm{mg} / \mathrm{kg} /$ day for 2 consecutive days). As shown in Figure 2 and Table 1, serum content of cTnI and kinase activities of CK, CK$\mathrm{MB}, \mathrm{LDH}$, and $\alpha-\mathrm{HBD}$ were increased in the ISO group (positive myocardial injury model), and decreased in MET group (positive drug treatment) (vs. control, $P<0.05$ ), which demonstrated that the ISO-induced myocardial injuries were stable and replicable. Moreover, all indexes of myocardial injury (cTnI, CK, CK-MB, LDH, and $\alpha$-HBD) in the STDP group were decreased (vs. ISO, $P$ $<0.05$ ), which showed that STDP alleviated the ISOinduced myocardial injury in vivo.

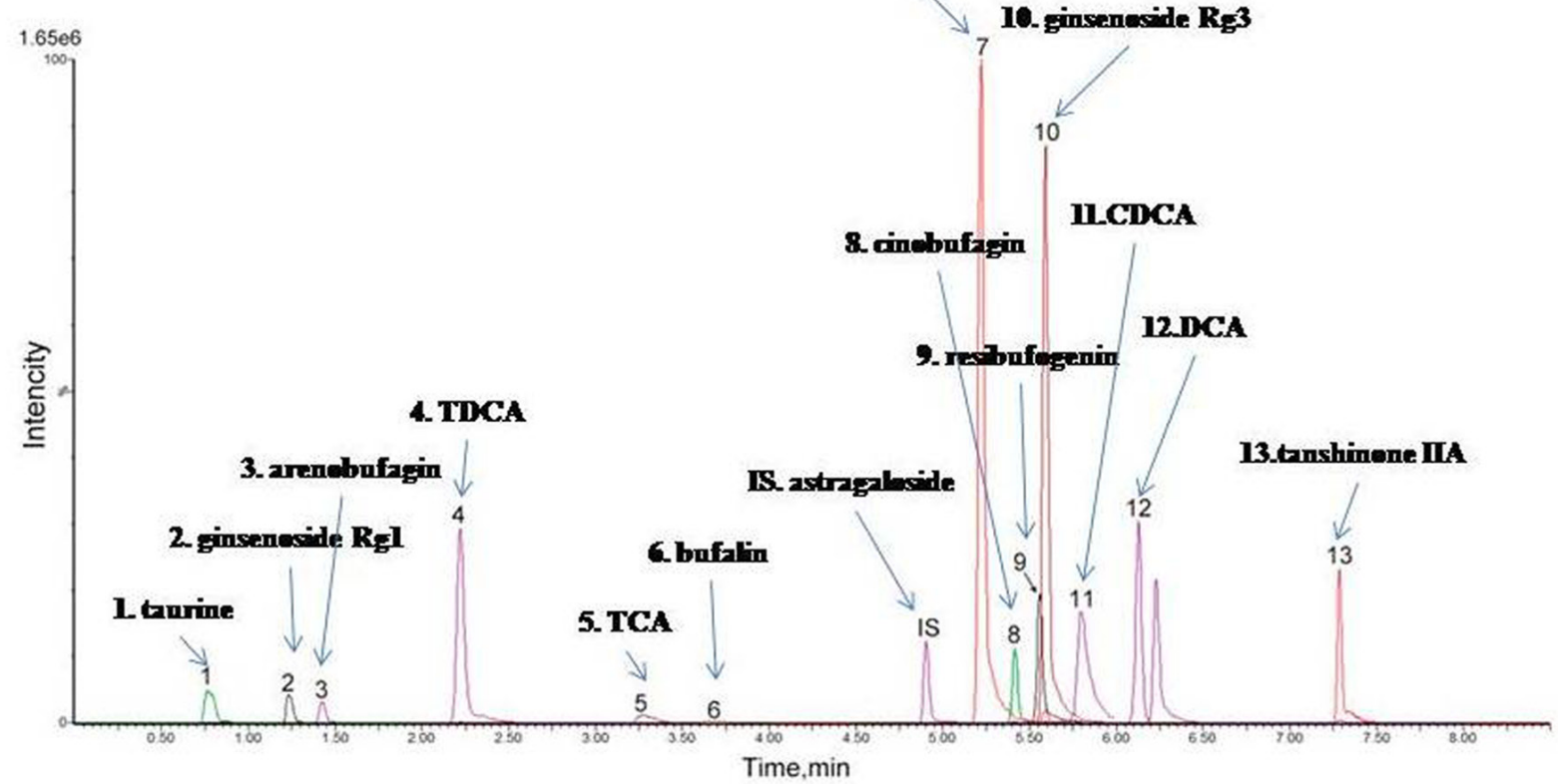

Figure 1: Thirteen major chemical constituents in STDP. MRM chromatograms of 13 markers and internal standard: (1) taurine, (2) ginsenoside Rg1, (3) arenobufagin, (4) TDCA, (5) TCA, (6) bufalin, (7) CA, (8) cinobufagin,(9) resibufogenin, (10) ginsenoside Rg3, (11) CDCA, (12) DCA, (13) tanshinone IIA,(IS) astragaloside. 
Table 1: Elisa data of four groups

\begin{tabular}{|c|c|c|c|c|}
\hline & $\mathrm{CON}$ & ISO & MET & STDP \\
\hline cTnI(pg/ml) & $0.335 \pm 0.06$ & $1.900 \pm 0.71^{*}$ & $0.322 \pm 0.06^{\#}$ & $0.403 \pm 0.05^{\#}$ \\
\hline CK(u/l) & $422.4 \pm 36.0$ & $3140 . \pm 686^{* * * *}$ & $1322 . \pm 261^{\#}$ & $900.4 \pm 175.1^{\# \#}$ \\
\hline CK-MB(u/l) & $383.8 \pm 41.9$ & $545.9 \pm 31.7^{*}$ & $330.6 \pm 46.7^{\# \#}$ & $311.4 \pm 29.40^{\ldots \#}$ \\
\hline $\operatorname{LDH}(\mathbf{u} / \mathbf{l})$ & $256.8 \pm 16.9$ & $375.7 \pm 32.9^{*}$ & $297.5 \pm 42.1$ & $262.5 \pm 26.50^{\#}$ \\
\hline$\alpha-\operatorname{HBD}(u / \mathbf{l})$ & $65.14 \pm 9.20$ & $128.9 \pm 19.1^{*}$ & $122.3 \pm 22.3$ & $70.13 \pm 11.63^{\#}$ \\
\hline $\operatorname{AST}(\mathbf{u} / \mathbf{l})$ & $73.00 \pm 3.99$ & $129.4 \pm 24.9$ & $128.5 \pm 17.8$ & $90.00 \pm 10.26$ \\
\hline
\end{tabular}

Note: ${ }^{*} P<0.05,{ }^{* * *} P<0.001$, compared with the control group $(\mathrm{CON}) .{ }^{\#} P<0.05,{ }^{\# \#} P<0.01$, ${ }^{\# \#} P<0.001$, compared with the ISO group.
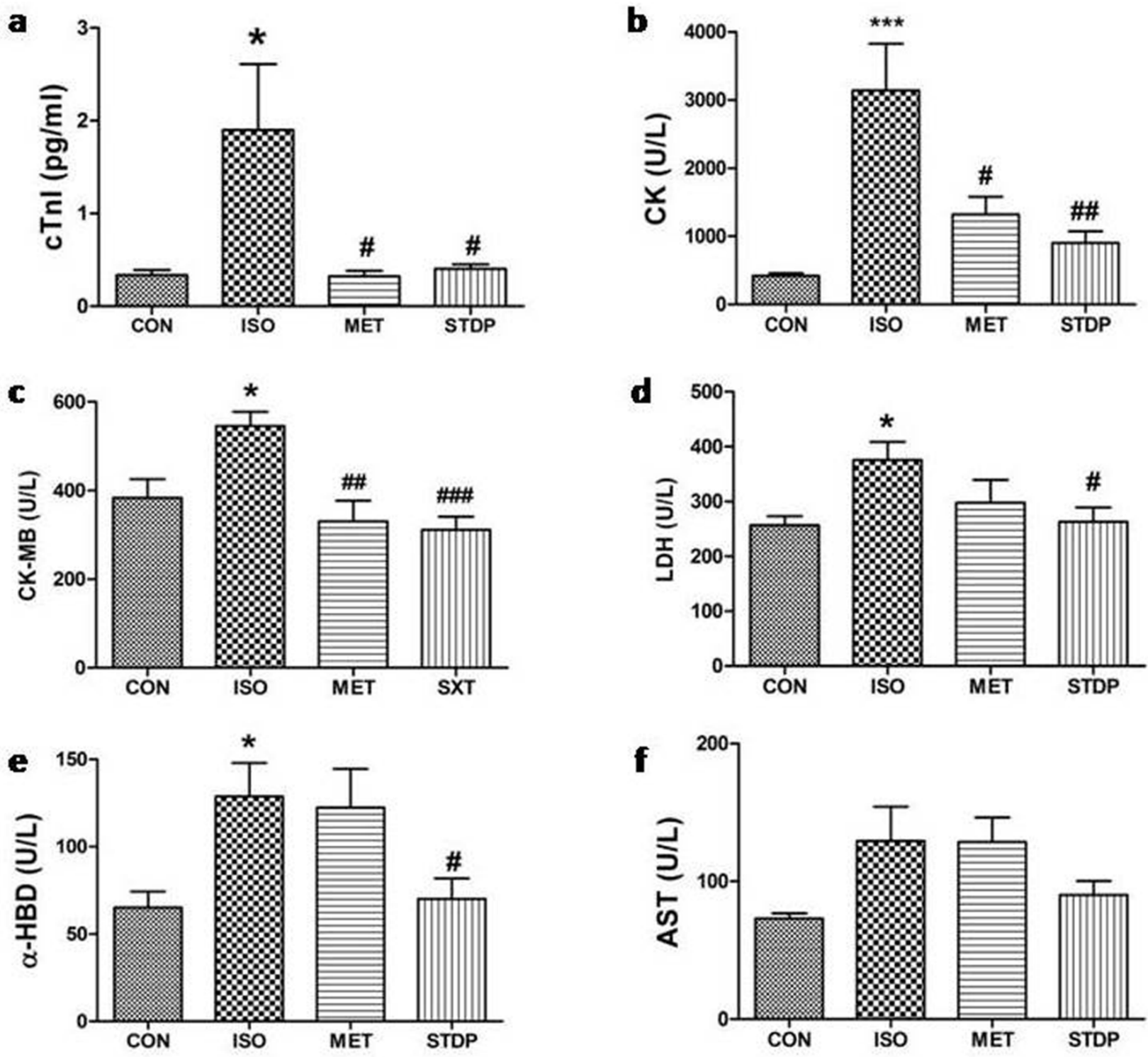

Figure 2: Effects of STDP on serum indices of myocardial injury in rats. Plasma levels of (a) cTnI, (b) CK, (c) CK-MB, (d) $\mathrm{LDH}$, (e) $\alpha$-HBD, (f) AST among the4 study groups (CON, ISO, MET, and STDP) were measured by ELISA methods. ${ }^{*} \mathrm{P}<0.05,{ }^{* * *} \mathrm{P}<$ 0.001, compared with CON; ${ }^{\mathrm{P}}<0.05$, ${ }^{\#} \mathrm{P}<0.01$, "\# $\mathrm{P}<0.001$, compared with the ISO group. 
Subsequently, rats were sacrificed and their heart, lung, and liver were harvested and weighted (Figure 3, Table 2, and Supplementary Figures 1-2). Compared with the CON group, there were significantly increases in heart weight (HW), ratio of heart weight/body weight (HW/ $\mathrm{BW})$, and ratio of heart weight/tibial length $(\mathrm{HW} / \mathrm{TL})$ in the ISO group $(P<0.001)$, which confirmed that cardiac hypertrophy was formed after ISO treatment. Lung weight, ratio of Lung W/BW, and Lung W/TL were increased (vs. control, $P<0.05$ ), suggesting that lung edema was formed after ISO stimulation. Liver weight, ratio of Liver W/BW, and Liver W/TL were increased (vs. control, $P<0.05$ ), suggesting that right heart failure was formed in the ISO group. Thus, pathological cardiac remodeling was formed after ISO stimulation. Compared with the ISO group, STDP and MET group did not have significant differences
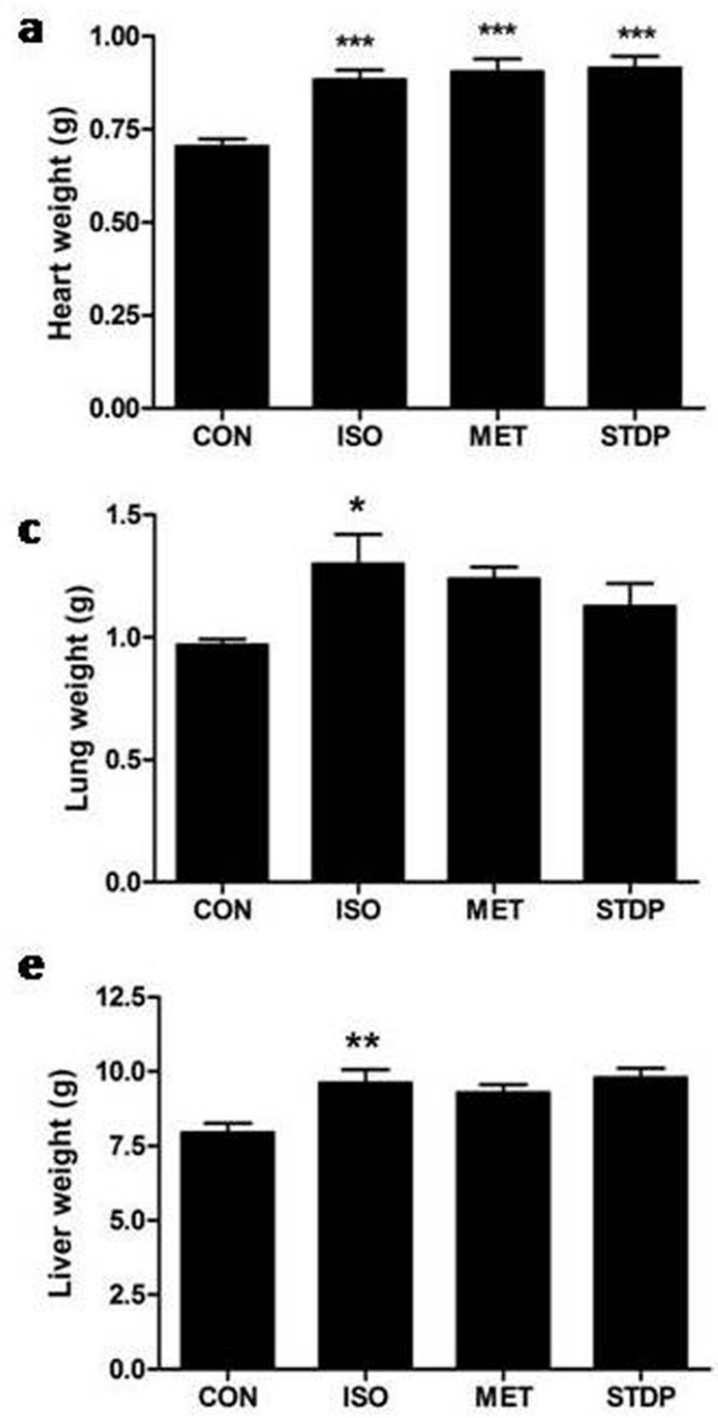

in cardiac hypertrophy. However, BNP were significantly reduced in the STDP group than ISO group $(P<0.05$, Supplementary Figure 3), which suggested that STDP partly alleviated acute pathological cardiac remodeling.

\section{STDP alleviated myocardial ischemic injury dose-dependently in rats}

To determine dose-response relationship of the effects of STDP on ISO-induced myocardial ischemic injury in rat, an ELISA method was implemented and cTnI was chosen as the marker of myocardial injury due to its high accuracy, super sensitivity and high specificity. Five doses of STDP $\left(1,10,100,1000\right.$, and $10000 \mathrm{mg} \cdot \mathrm{kg}^{-}$ ${ }^{1} \cdot$ day $^{-1}$ ) were selected. As shown in Figure 4a. myocardial injury was not reduced by 1 or $10 \mathrm{mg} / \mathrm{kg}$ /day STDP, while
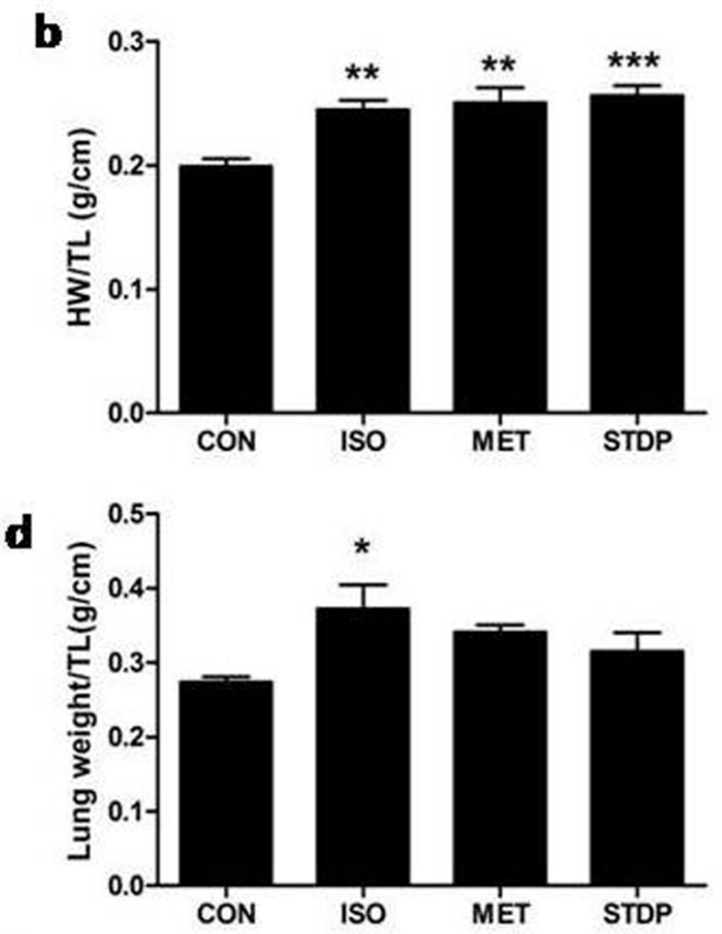

f

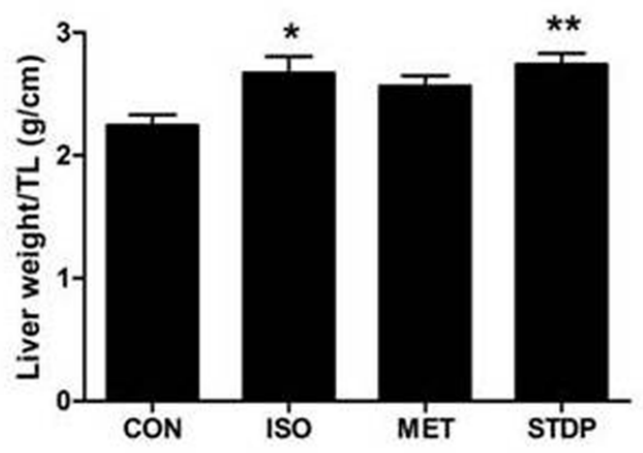

Figure 3: Anatomic data among the four groups. (a) Heart weight (HW), (b) Ratio of HW/TL, (c) Lung weight, (d) Ratio of Lung weight/TL, (e) Liver weight, (f) Ratio of Liver weight/TL were measured. Data are presented as mean \pm S.E.M. ${ }^{*} \mathrm{P}<0.05$, ${ }^{* *} \mathrm{P}<0.01,{ }^{* * *}$ $\mathrm{P}<0.001$ compared with the CON group. 
Table 2: Anatomical data of four groups

\begin{tabular}{lcccc}
\hline & CON & ISO & MET & STDP \\
\hline BW(g) & $217.9 \pm 4.35$ & $210.9 \pm 4.73$ & $210.8 \pm 5.74$ & $211.6 \pm 1.82$ \\
Heart(g) & $0.705 \pm 0.02$ & $0.884 \pm 0.03^{* * *}$ & $0.904 \pm 0.04^{* * *}$ & $0.916 \pm 0.03^{* * *}$ \\
Lung(g) & $0.969 \pm 0.02$ & $1.299 \pm 0.12^{*}$ & $1.238 \pm 0.05$ & $1.127 \pm 0.09$ \\
Liver(g) & $7.946 \pm 0.31$ & $9.621 \pm 0.45^{* *}$ & $9.291 \pm 0.27$ & $9.786 \pm 0.32^{* *}$ \\
TL(cm) & $3.542 \pm 0.03$ & $3.609 \pm 0.03$ & $3.624 \pm 0.05$ & $3.572 \pm 0.02$ \\
HW/BW & $3.234 \pm 0.06$ & $4.201 \pm 0.15^{* * *}$ & $4.319 \pm 0.26^{* * *}$ & $4.323 \pm 0.12^{* * *}$ \\
Lung/BW & $4.456 \pm 0.12$ & $\mathbf{6 . 2 2 0} \pm 0.66^{*}$ & $5.868 \pm 0.14$ & $5.325 \pm 0.43$ \\
Liver/BW & $36.51 \pm 1.30$ & $45.53 \pm 1.54^{* *}$ & $44.31 \pm 2.13$ & $46.29 \pm 1.57^{* * *}$ \\
HW/TL(g/cm) & $0.199 \pm 0.01$ & $0.245 \pm 0.01^{* *}$ & $0.250 \pm 0.01^{* * *}$ & $0.256 \pm 0.01^{* * *}$ \\
Lung/TL(g/cm) & $0.274 \pm 0.01$ & $0.373 \pm 0.03^{*}$ & $0.341 \pm 0.01$ & $0.315 \pm 0.03$
\end{tabular}

Note: ${ }^{*} P<0.05,{ }^{* *} P<0.01,{ }^{* * *} P<0.001$, compared with the control group (CON).

significantly inhibited by $100 \mathrm{mg} / \mathrm{kg} /$ day STDP. Over $100 \mathrm{mg} / \mathrm{kg} /$ day, STDP protected against ISO-induced myocardial injury with statistical significance $(P<0.05)$. The dose-response curve followed a sigmoidal shape (Figure 4b) with a half-maximal response at $42 \mathrm{mg} \cdot \mathrm{kg}$ ${ }^{1}$ day $^{-1}$. Therefore, STDP protected against the ISOinduced myocardial injury dose-dependently in rats.

\section{Phosphorylation of ERK1/2 was increased in ischemic cardiac tissue in vivo and in vitro}

To investigate potential mechanisms of STDP protection on myocardial ischemic injury, we studied three major signaling pathways in myocardial ischemic injury, ERK1/2, AKT, and CaMKII [9-12]. Some researchers reported that the phosphorylation of ERK1/2 was increased in ischemic myocardium and played a harmful effect, while others reported that ERK1/2 played a protective role in the RISK pathway [13-16]. Therefore, it is hard to differentiate that the phosphorylation of ERK $1 / 2$ was beneficial and/or harmful to ischemic myocardium. In the present study, Western blot analysis showed that phophsrylations of ERK1/2, AKT, and CaMKII were all enhanced after ISO stimulation (vs. $\mathrm{CON}, P<0.05$, Figure 5). Furthermore, phosphorylation of ERK1/2, AKT, and CaMKII were inhibited after metoprolol precubation (MET vs ISO group, $\mathrm{P}<0.05$ ) in consistence with previous publications and suggested stability and reproducibility of
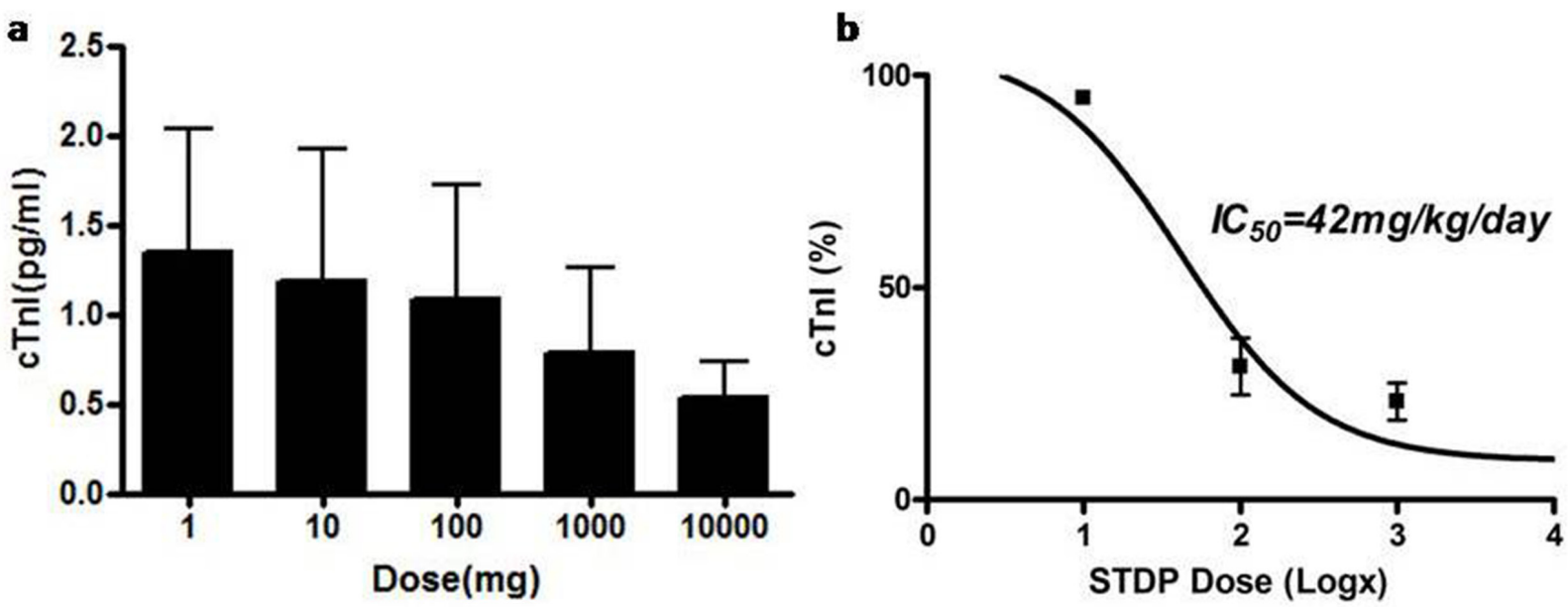

Figure 4: STDP inhibited serum cTnI expression dose-dependently in rats. (a) cTnI were detected in different STDP concentrations from 1 to $10000 \mathrm{mg} / \mathrm{kg}$ /day by ELISA analysis. (b) Dose-dependent curve was obtained to normalize the inhibitive effect of STDP on rats' myocardial ischemic injury, IC50 $=42 \mathrm{mg} / \mathrm{kg} /$ day. 
our ISO-induced myocardial injury model. Interestingly, phosphorylations of ERK1/2 were significantly increased in the STDP group, (vs. ISO group, $P<0.05$ ), whereas both AKT and CaMKII phosphorylations were not significantly changed (vs. ISO group, $\mathrm{P}>0.05$ ). Together, these data demonstrated activation of ERK1/2 signaling pathway, but not AKT and CaMKII, by STDP stimulation in its protection against myocardial ischemic injury.

To further validate the in vivo experiments, in vitro rat $\mathrm{H} 9 \mathrm{C} 2$ cardiomyocytes were cultured to simulate ischemia injury by ISO pre-incubation $(1 \mu \mathrm{M}$ ISO for 1.5 hours, Figure 6) followed by metoprolol or STDP stimulation. Western blot analysis showed enhancement of ERK1/2 phosphorylation after STDP stimulation (vs. ISO group, $P<0.05$, Figure $6 \mathrm{a}-6 \mathrm{~b}$ ). The phosphorylations of AKT and CaMKII were not changed significantly (vs. ISO group, $P>0.05$, Figure $6 \mathrm{c}-6 \mathrm{~d})$, consistent with the in vivo data in rat cardiac tissue. Together, both in vivo and in vitro data supported that STDP activated ERK1/2 phosphorylation so as to protect against ISO-induced myocardial ischemic injury.

PD98059, a ERK1/2 inhibitor, attenuated the protective effects of STDP in ischemic ischemia tissue in rats

To confirm the role of ERK1/2 phosphorylation in STDP's protective effects against the ISO-induced myocardial ischemic injury, we administrated PD98059 (a ERK1/2 inhibitor, PD), LY294002 (a PI3-K/AKT inhibitor, LY), and KN-93 (a CaMKII inhibitor, KN) i.h, after ISO and STDP stimulation. As shown in Figure 7, cTnI was increased in the ISO group and decreased in MET and STDP groups, in consistence with our previous results. However, cTnI in the PD group were significantly increased compared with STDP group $(P<0.05)$, and no significantly change compared with ISO group $(P$ $>0.05)$, which suggested that the STDP's protection

\section{Tissue}

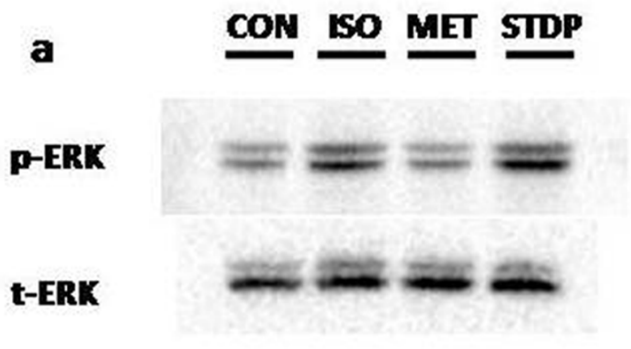

42
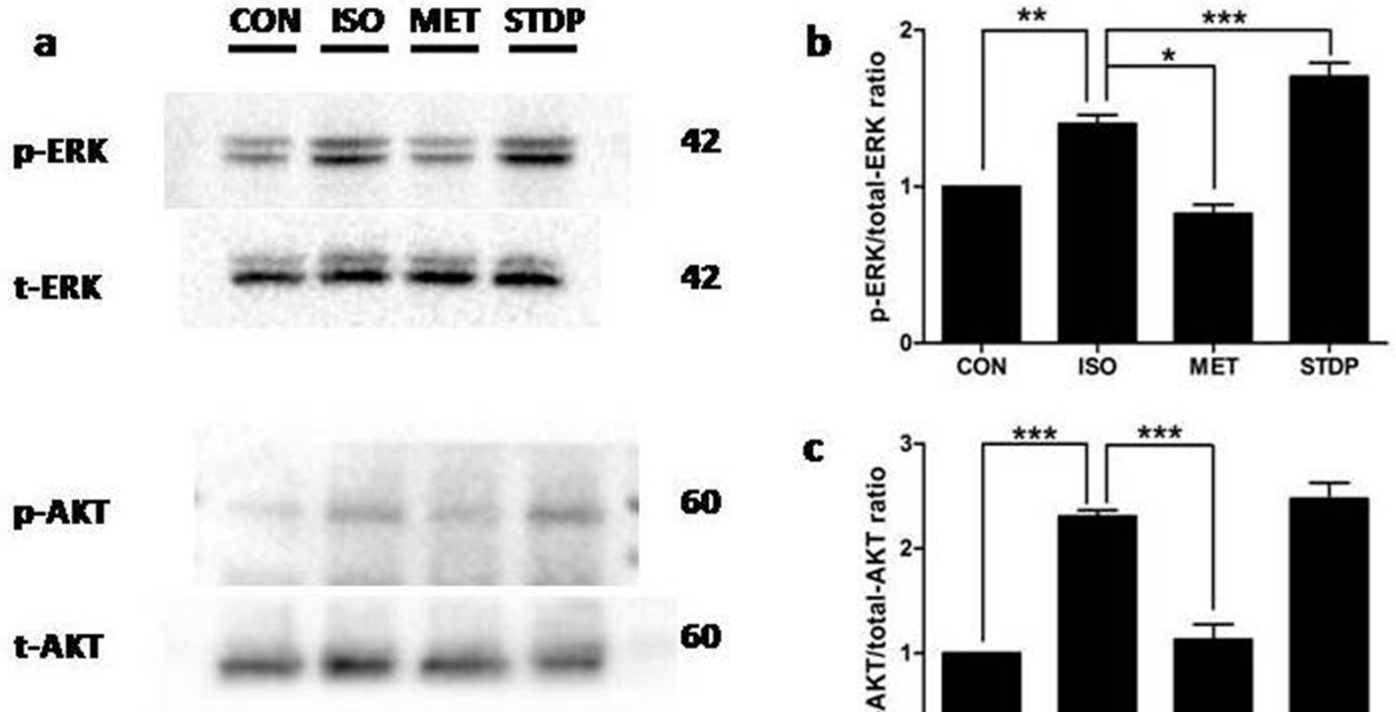

60
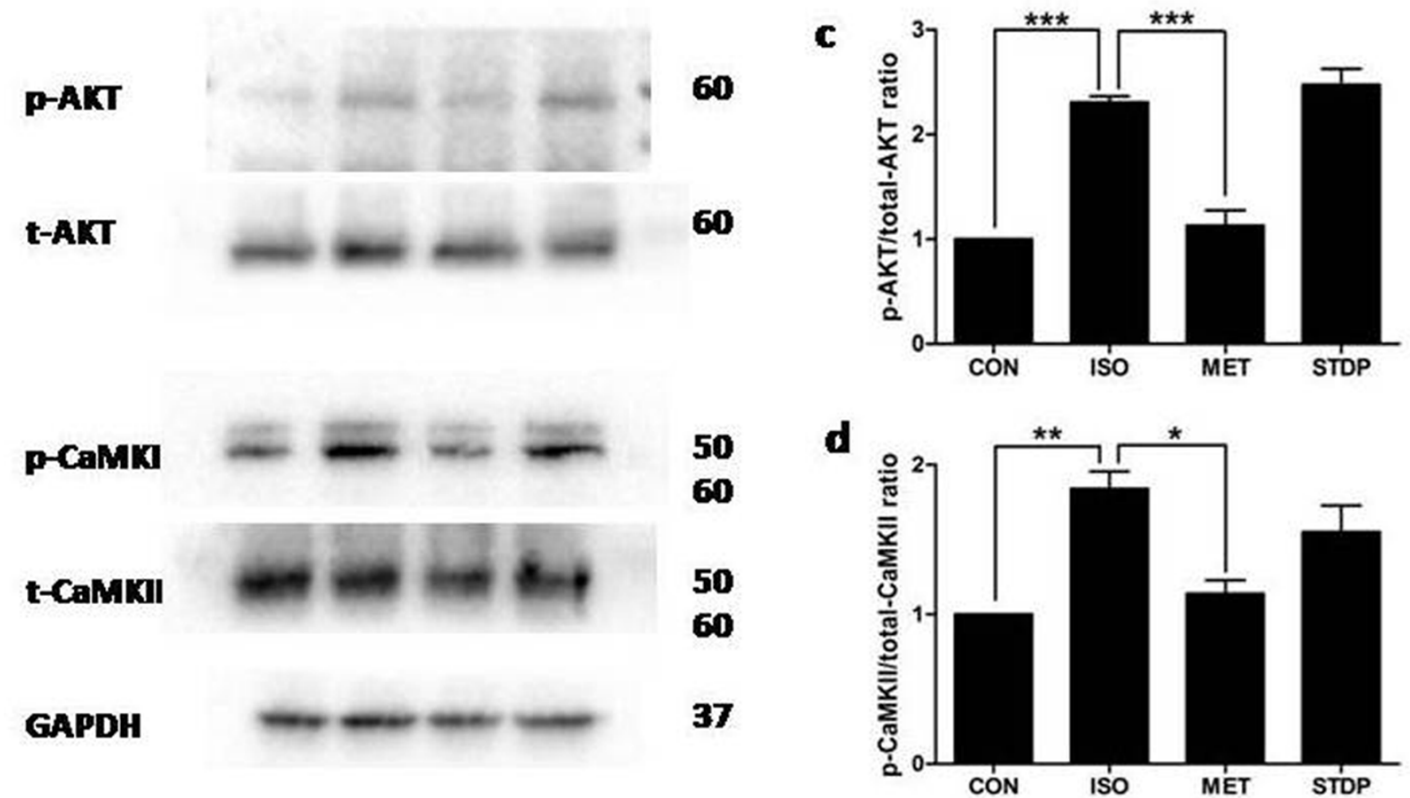

Figure 5: STDP enhanced ERK1/2 phosphorylation after ISO stimulation in vivo. (a) Western blotting was performed to detect phosphor-ERK1/2, AKT, and CaMKII. Total proteins and GAPDH were used as loading controls. (b) p-ERK1/2, (c) p-AKT, (d) $\mathrm{p}$-CaMKII (mean \pm SEM, $\mathrm{n}=3$ ) were expressed as fold changes from phosphorylated and total proteins. ${ }^{*} \mathrm{P}<0.05,{ }^{* *} \mathrm{P}<0.01,{ }^{* * *} \mathrm{P}<0.01$. 
diminished after inhibiting the activation of ERK1/2 (PD group) whereas STDP protective effects was still exist even after KN and LY intervention $(P<0.05)$. Thus, both pharmacological and molecular methods supported the conclusion that STDP protected against myocardial injury via an ERK1/2-dependent signaling pathway.

Based on the above observations, we formulated following work model (Figure 8): The ISO-induced myocardial ischemia was associated with enhanced phosphorylations of ERK1/2, AKT, and CaMKII. STDP further activated the ERK1/2 phosphorylation, but neither AKT nor CaMKII, to protect against ISO-induced myocardial ischemic injury.

\section{DISCUSSION}

This is the first study to investigate the dosedependent protective effects of STDP against myocardial ischemia injury in rat. We demonstrated that ERK1/2 inhibition by PD98059 abolished the STDP-induced cardio-protection, suggesting that STDP's protection against myocardial ischemic injury was via an ERK1/2dependent signaling pathway. These studies provided direct experimental support to STDP application in clinics and hospitals.

We constructed myocardial ischemic injury model by ISO i.h administration in rats. High dose ISO induced pathological changes, such as hypoxia, necrosis, and inhibition of cardiac function, similar to the changes in the AMI region of human heart [17]. As shown in Figure 2, cTnI, CK, CK-MB, LDH, and $\alpha-\mathrm{HBD}$ were increased in the ISO-administrated rats. Patel et al. [18] reported that the ISO-induced cardiac pathological remodeling in rats might be due to the increased water content, edematous intramuscular space and extensive necrosis of inflammatory cardiac muscle. STDP inhibited the ISOinduced myocardial injury and reversed pathological cardiac remodeling, which further supported the role of

\section{Cell}

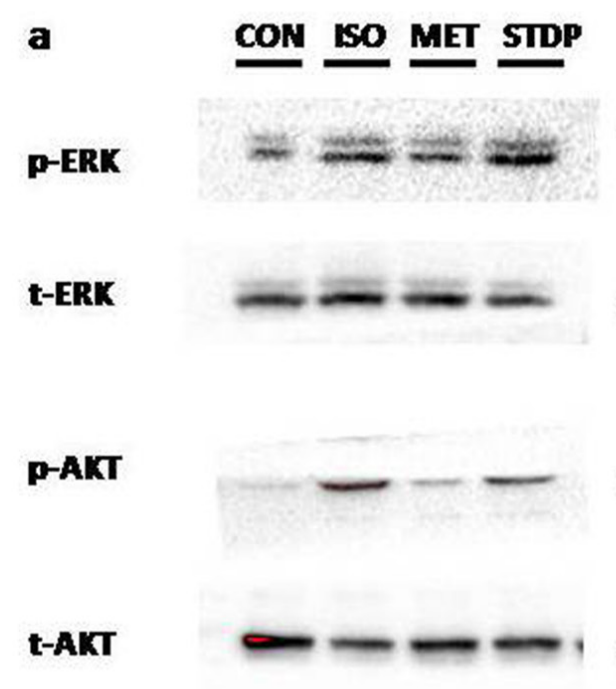

42

42

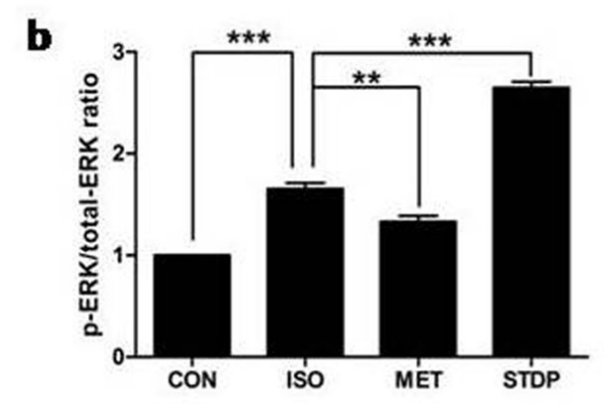

60
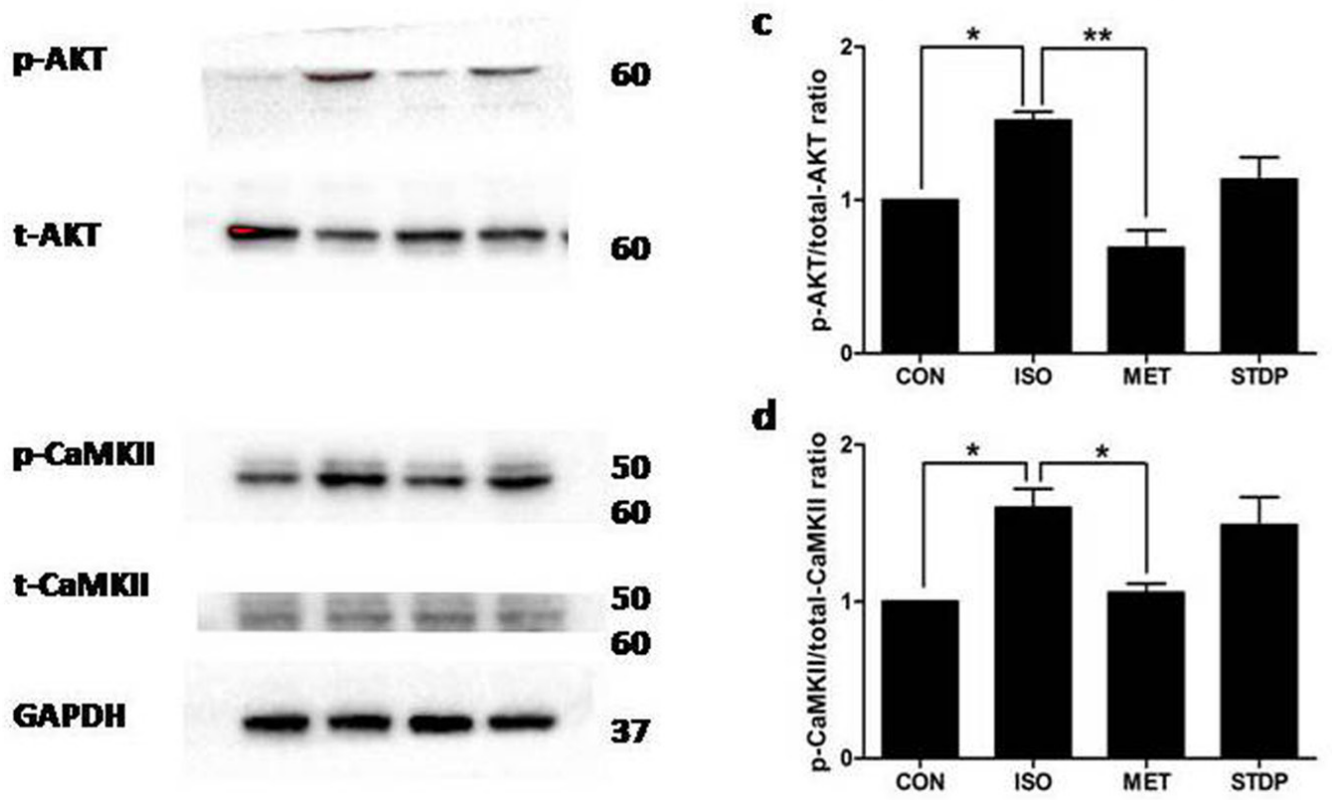

Figure 6: STDP enhanced ERK1/2 phosphorylation in vitro. (a) Western blotting was performed to detect phosphor-ERK1/2, AKT, and CaMKII. Total proteins and GAPDH were used as loading controls. (b) p-ERK1/2, (c) p-AKT, (d) p-CaMKII (mean \pm SEM, $\mathrm{n}=$ 3) were expressed as fold changes from phosphorylated and total proteins. ${ }^{*} \mathrm{P}<0.05,{ }^{* *} \mathrm{P}<0.01,{ }^{* * *} \mathrm{P}<0.01$. 


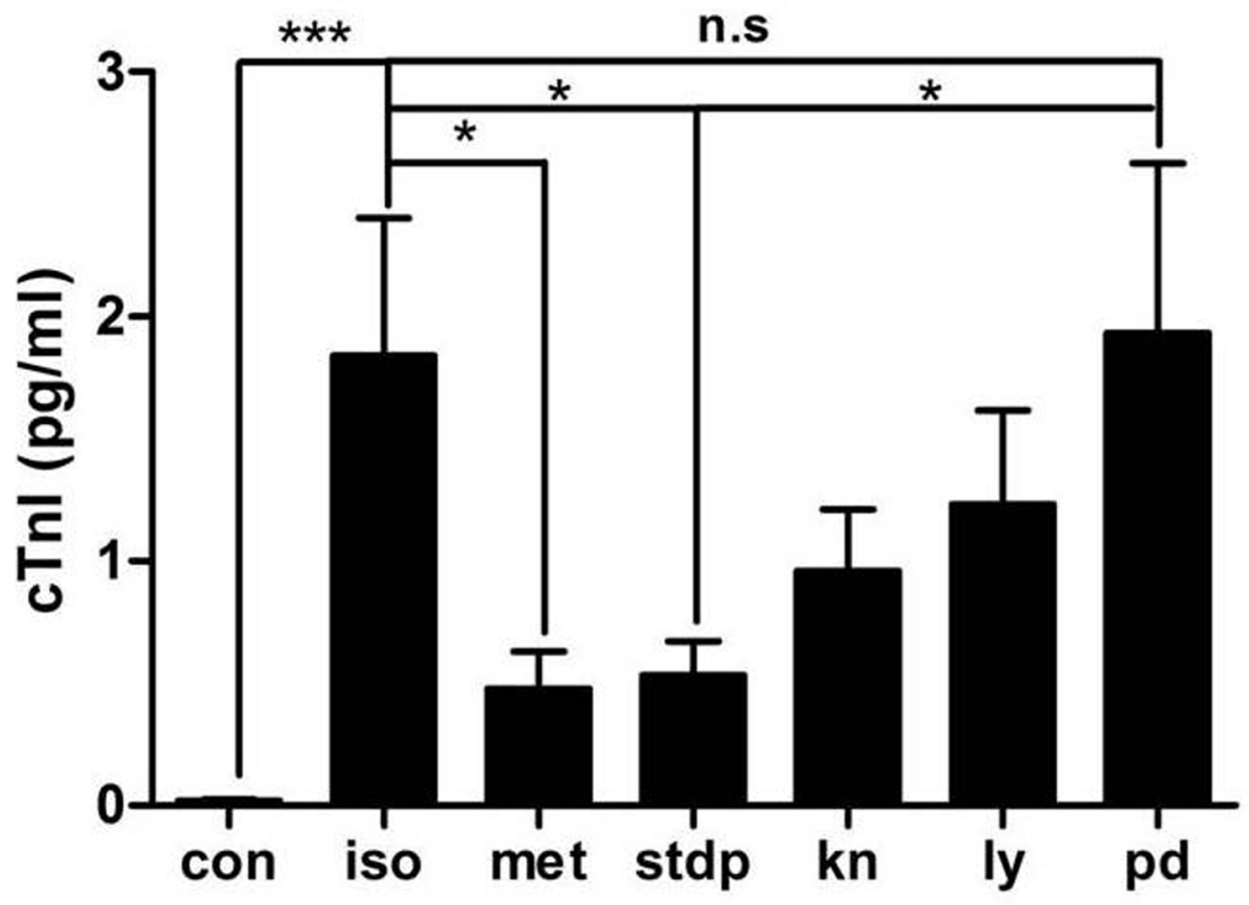

Figure 7: The protective effect of STDP on ISO-induced myocardial ischemic injury was diminished after PD98059 stimulation in vivo. Plasma levels of cTnI among the 7 study groups (CON, ISO, MET, STDP, KN, LY, and PD) were measured by ELISA methods. ${ }^{*} \mathrm{P}<0.05,{ }^{* *} \mathrm{P}<0.01,{ }^{* * *} \mathrm{P}<0.001$, n.s.: no significant difference.

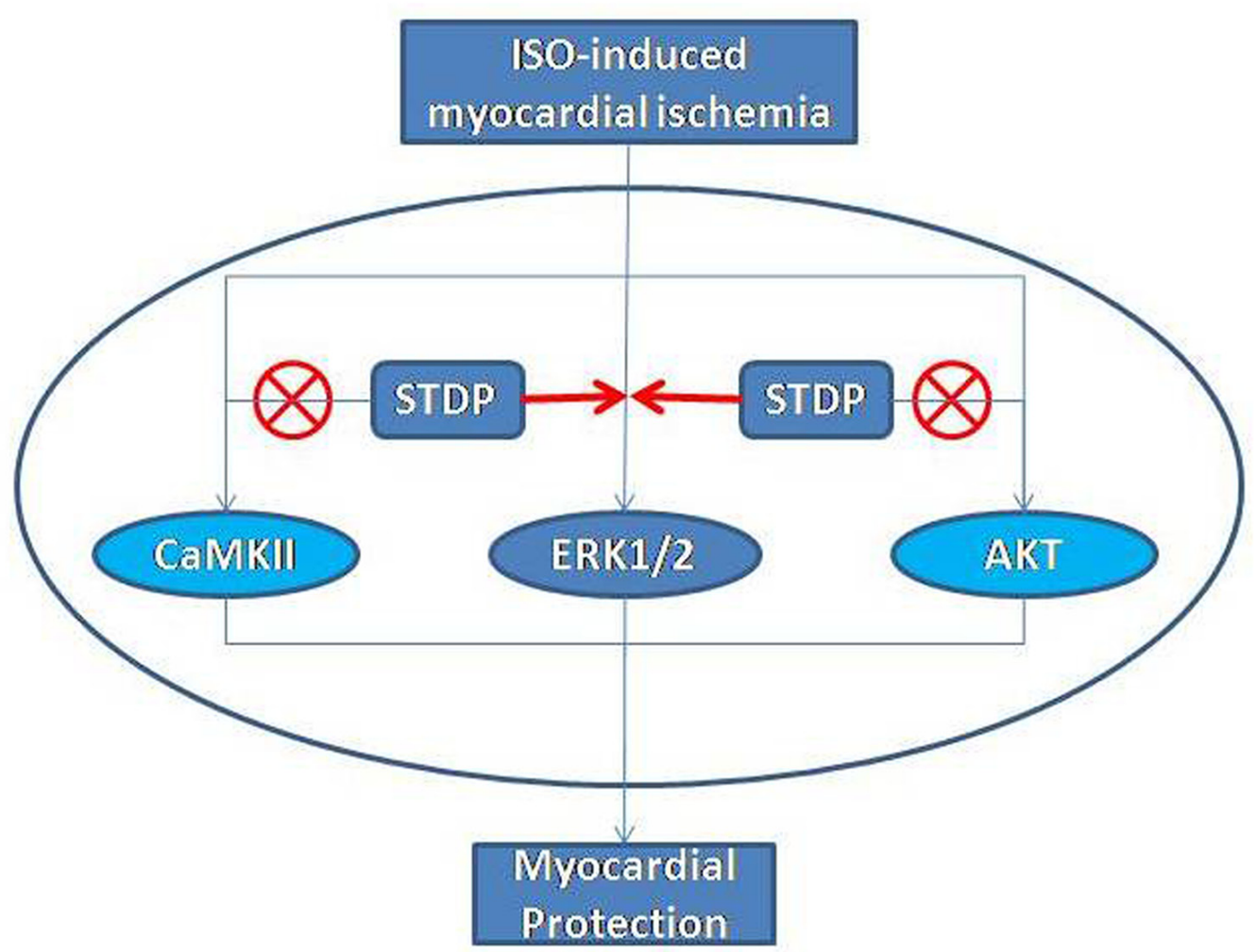

Figure 8: A model of pathways in the cardio-protection of STDP against ISO-induced myocardial ischemic injury. Isoproterenol induced myocardial ischemic injury in rats. STDP enhanced the phosphorylation of ERK1/2, but neither AKT nor CaMKII signaling pathway, leading to decrease myocardial injury. $\otimes$ Denotes non-participation of the signaling pathways. 
STDP in protecting heart against myocardial ischemic injury.

Mechanisms of myocardial ischemia injury are complex. One of the most classical protective mechanisms of ischemic injury is RISK signaling pathway. ERK1/2 and AKT proteins were shown to play key roles in the RISK pathway [15]. It was reported that the phosphorylation of ERK1/2 was increased in ischemic myocardium, leading to cardiomyocytes apoptosis, and thus, played a harmful effect in the process of myocardial ischemia [13, 14]. More publications supported that ERK1/2 protein played a key role in the RISK pathway and was one of the most classical protective mechanisms of ischemic injury [15, 16]. In addition, it was reported that bidirectional control of ERK1/2 function in the pituitary provided a key mechanism for endocrine gene control [19]. Therefore, it is not as simple to label either a beneficial or harmful role of the phosphorylation of ERK1/2 to ischemic myocardium. In the present study, our data indicated that the phosphorylation of ERK1/2 was harmful in a lower expression while played a protective role in a relative higher expression, thus, bidirectional controls of ERK1/2 function in the ischemic myocardium in rats.

Recently, CaMKII was studied as a vital molecule in pathological cardiac remodeling $[20,21]$. Considering pharmacological inhibition of CaMKII and activation of the ERK1/2 and PI3K/AKT protected cardiomyocytes from apoptosis during simulated ischemia in vitro, we chose these three pathways as the main targets in our study $[22,23]$. Although CaMKII and PI3-K/AKT signaling activation were reported to be important, our present results showed that STDP treatment had little influence on the CAMKII and PI3K/AKT signaling pathways (Figures 5-7), while STDP treatment promoted the phosphorylation of ERK1/2 after ischemia. These results suggested that ERK1/2 mediated the STDP-induced cardioprotection against acute myocardial ischemia injury, in consistence with previous reports (Figure 6) [16]. Therefore, our findings contributed to understanding the molecular mechanisms of cardiac protection of STDP.

With more than 2 decades of clinical applications, clinical pharmacological studies have demonstrated STDP's effects, including anti-AS, improving microenvironment, and protecting myocytes and vascular endothelial cells [24]. Moreover, long-term STDP intake was reported to significantly improve heart function and decrease angina pectoris attack as well as protect patients from myocardial infarction [25]. Our study showed that STDP had a dose-dependent inhibitive effect on the elevation of cTnI, CK, CK-MB, LDH, and $\alpha-\mathrm{HBD}$ during myocardial ischemia.

Since there are 13 major active ingredients in STDP, one may possibly postulate that STDP could work on multiple mechanisms of myocardial ischemia simultaneously. It was reported that STDP could reduce pituitrin induced acute myocardial ischemia via an anti - apoptotic signaling pathway, down-regulating expression of $\mathrm{Bax}$ and up-regulating expression of $\mathrm{Bcl}-2$ in myocardial tissue [26]. Moreover, STDP was reported to attenuate atherosclerotic lesions in ApoE(-/-) mouse model via reducing the aortic expression of miR-21a, miR-132, miR-126a, miR-155 and increasing expression of miR-20a [16]. However, numerous studies showed that Compound Chinese Medicine could take one aspect effect through a single signaling pathway, such as Wenxin Keli, Salvionate, and Tongxinluo capsule [27-29]. In the present study, it was showed that STDP worked via a single signaling pathway, in consistence with these publications [27-29]. Thus, this study provided a mechanism for the clinical STDP treatment in improving cardiac function of angina pectoris patients.

To supplement molecular studies on the mechanisms we used pharmacological inhibitors. However, these inhibitors might have limited specificity and completeness in blocking their target proteins. Further studies combining pharmacological and genetic evidences may be needed to supports our hypothesis that ERK $1 / 2$ is a key downstream molecular mediator of STDP in the RISK signaling pathway.

In summary, the present study showed that STDP reduced the ISO-induced myocardial ischemic injury. This result experimentally proved the effectiveness of STDP as a clinical therapy to protect against ischemia injury at an early stage. These experimental evidences of the STDPinduced cardio-protection can help to explain the improved outcomes in patients with acute coronary syndrome treated with STDP and may lead to the development of better drugs and/or new therapeutic applications of STDP.

\section{MATERIALS AND METHODS}

\section{Animals and reagents}

This study was performed in accordance with the guidelines and with approval from the Institutional Animal Care and Use Committee of Guangdong Province Hospital of Chinese Medicine, Guangzhou University of Traditional Chinese Medicine, and with the Guide for the Care and Use of Laboratory Animals (Department of Health and Human Services, National Institutes of Health, Publication No. 86-23, revised 1996).

10-12 weeks male wild-type SD rats $(200 \pm 5 \mathrm{~g}$ body weight) were obtained from the Experimental Animal Center of Guangdong Province. STDP was provided by Inner Mongolia Conba Pharmaceutical Co., Ltd. (Inner Mongolia, China).

\section{Rat myocardial ischemic injury model in vivo}

A model of myocardial ischemic injury was constructed by isoproterenol (ISO, $85 \mathrm{mg} / \mathrm{kg} /$ day for 2 days) subcutaneous administration (i.h) in rats. Serum 
levels of cardiac troponin I (cTnI), creatine kinase (CK), CK-MB, lactate dehydrogenase (LDH), aspartate transaminase (AST), $\alpha$-hydroxybutyric dehydrogenase $(\alpha-\mathrm{HBD})$ were detected. Rats were assigned to four groups: CON $(n=9)$, ISO $(n=8)$, MET $(n=6)$, and STDP $(n=9)$. Rats in CON group received saline i.p and all the procedures except ISO administration; rats in ISO group were subjected to ISO administration (i.p) for 2 days; MET rats received metoprolol, $10 \mathrm{mg} / \mathrm{kg} /$ day i.p, for 3 days prior to and 2 days after ISO stimulation (totally 5 days), to serve as positive controls of the protective effects of ischemia treatments. Based on our previous study, literature, and clinical usage in patients (with dose conversion between humans oral usage and animals), STDP powder at a dose of $3 \mathrm{~g} / \mathrm{kg}$ body weight mixed with $1 \mathrm{~mL}$ saline was administered daily via direct gastric gavage, for 3 days prior to and 2 days after ISO stimulation (totally 5 days).

\section{ELISA analysis}

At the time of sacrifice (the day after ISO i.p for 2 days) and under anesthesia, $1 \mathrm{ml}$ of blood was collected from the inferior vena cava of each rat and immediately centrifuged at 3000 revolutions per minute for 15 minutes. The plasma supernatant was recovered and kept at $-80^{\circ} \mathrm{C}$ until performing ELISA assays of cTnI, CK, CK-MB, AST, $\mathrm{LDH}$, and $\alpha$-HBD concentrations at $25^{\circ} \mathrm{C}$ on a Hitachi 7180 analyzer. Following assays were performed according to manufacturer's protocols: CK ELISA kit (Ref\#12132672), CK-MB ELISA kit (Ref\#12132893), cTnI high sensitive ELISA kit (Ref\#05092728), AST ELISA kit (Ref\#11876848), LDH ELISA kit (Ref\#03002209), and $\alpha$-HBD ELISA kit (Ref\#11876937) (Roche Diagnostics, Indianapolis, IN, USA).

\section{Dose-dependent experiment of cTnI in vivo}

To evaluate the dose-dependent effect of STDP on ISO-induced myocardial ischemia in rats, five concentrations of $\operatorname{STDP}(1,10,100,1000$ and 10000 $\mathrm{mg} \cdot \mathrm{kg}-1 \cdot$ day-1, respectively) were used in 5 separate animal groups: STDP-1 mg group $(n=9)$, STDP-10 mg group $(n=9)$, STDP-100 mg group $(n=9)$, STDP-1000 mg group $(n=9)$, and STDP-10000 mg group $(n=6)$. cTnI concentrations was detected as a golden indicator of myocardial injury by using a cTnI high sensitive ELISA kit on a Hitachi 7180 analyzer. Dose-response curve of DLT was analyzed.

\section{Rat H9C2 cardiomyocyte culture in vitro}

Rat H9C2 cardiomyocyte cell line was obtained from the American Type Culture Collection (ATCC, Manassas, VA, USA). H9C2 cells were seeded in 6-well plates, and maintained in DMEM supplemented with $10 \%$ fetal calf serum at $37^{\circ} \mathrm{C}$ in $\mathrm{CO}_{2}$ incubation. The medium was replaced every $2-3$ days, and cells were sub-cultured or subjected to experimental procedures at $80-90 \%$ confluence. Subsequently, the H9C2 cells were synchronized in serum-free DMEM $24 \mathrm{~h}$ before treatment. To evaluate cardio-protective effects of STDP on rat $\mathrm{H} 9 \mathrm{C} 2$ cells, four groups were studied: Control, ISO, MET, and STDP. ISO group was subjected to $1 \mu \mathrm{M}$ ISO for 30 minutes while Control group was stimulated with $0.2 \mathrm{ul}$ DMSO. MET group (10uM, served as positive drug treatment $)$ and STDP group $(0.65 \mathrm{mg} / \mathrm{ml})$ were pretreated for 2 hours followed by ISO stimulation. After ISO preincubation for 30 minutes, all cell samples were lysed in $150 \mu \mathrm{l}$ cell lysis buffer for Western blot experiments. All experiments were performed in triplicate.

\section{Western blot analysis}

The cardiac tissue cells were rinsed and homogenized in RIPA lysis buffer containing protease inhibitor PMSF. The insoluble protein lysate was removed by centrifugation at $12,000 \mathrm{rpm}$ for $5 \mathrm{~min}$ at $4^{\circ} \mathrm{C} .50 \mu \mathrm{g}$ of the protein lysate was resolved using $12 \%$ SDS-polyacrylamide gel electrophoresis. The gels were transferred to polyvinylidene difluoride (PVDF) membranes by semidry electrophoretic transfer at 200 $\mathrm{mA}$ for $60 \mathrm{~min}$. The PVDF membranes were blocked one hour in 5\% milk at room temperature and subjected to Western blot analysis. Following antibodies were used: anti-phospho-ERK1/2 (Thr202/Tyr204), antiphospho-PKB (Ser473), anti-phospho-CaMKII, and anti-PKB (Cell Signaling Technology, Beverly, MA, USA), anti-ERK1/2, anti-GAPDH and anti-eIF-5 (Santa Cruz Technology, Delaware, CA, USA). The sheets were analyzed with antibodies according to the supplier's protocol, and visualized with peroxidase and an enhanced-chemiluminescence system (ECL kit, Pierce Biotechnology, Inc.). Bands were visualized by use of a super western sensitivity chemiluminescence detection system (Pierce, IL). Autoradiographs were quantitated by densitometry (Science Imaging system, Bio-Rad, Hercules, CA), and the ratio was compared between the phosphorylated p-ERK1/2, p-AKT, p-CaMKII and total proteins ERK1/2, AKT, CaMKII, respectively.

\section{Statistical analysis}

Data are reported as means \pm S.E.M. Bonferroni's post hoc method was used to assess the significance of differences using GraphPad Prism version 4.0. A P-value of $<0.05$ was considered statistically significant.

\section{Abbreviations}

STDP, Shexiang Tongxin dropping pill; ISO, isoproterenol; i.h, subcutaneous injection; ERK1/2, extracellular regulated protein kinases; PI3-K, phosphatidylinositol-3-kinase; CamKII, Ca2+/calmodulin- 
dependent protein kinase II; ELISA, enzyme linked immunosorbent assay; cTnI, cardiac troponin I; CK, creatine kinase; $\mathrm{CK}-\mathrm{MB}$, serum creatine kinase $\mathrm{MB}$; $\mathrm{LDH}$, lactate dehydrogenase; $\alpha-\mathrm{HBD}$, alpha-hydroxybutyrate dehydrogenase; AKT, protein kinase B; HW, heart weight; BW, body weight; TL, tibial length; IHD, ischemic heart disease; PD, PD98059; LY, LY294002; KN, KN-93.

\section{Author contributions}

Conceived and designed the experiments: M.Z., and J.Q. Performed the experiments: Y.T., J.L, D.F. Analyzed the data: J.Y., W.J., Contributed reagents/materials/ analysis tools: J.Q. Wrote the manuscript: J.Q.

\section{ACKNOWLEDGMENTS}

We acknowledge and appreciate our colleagues for their valuable efforts and comments on this paper.

\section{CONFLICTS OF INTEREST}

No conflicts of interest, financial or otherwise, are declared by the author(s).

\section{FUNDING}

This study was supported by the National Natural Science Foundation of China (81603429, 81473471), Natural Science Foundation of Guangdong Province(2014A030313402), Guangdong science and Technology Foundation (2016KT1170), Special Funds of Guangdong hospital of Chinese Medicine(YK2013B2N11, YN2014ZH01, YN2014ZHR203, YN2016QJ19).

\section{REFERENCES}

1. Benjamin EJ, Blaha MJ, Chiuve SE, Cushman M, Das SR, Deo R, de Ferranti SD, Floyd J, Fornage M, Gillespie C, Isasi CR, Jiménez MC, Jordan LC, et al. Heart disease and stroke statistics-2017 update: a report from the American Heart Association. Circulation. 2017; 135:e146-e603.

2. Gao XY, Zhou BY, Zhang MZ, Zhao X, Qing P, Zhu CG, Wu NQ, Guo YL, Gao Y, Li XL, Wang Y, Liu G, Dong Q, et al. Association between fibrinogen level and the severity of coronary stenosis in 418 male patients with myocardial infarction younger than 35 years old. Oncotarget. 2017; 8:81361-81368. https://doi.org/10.18632/oncotarget.18578.

3. Zhang J, Xu XM, Wang WM. ShexiangTongxin dropping pill combined with aspirin treatment of unstable angina. Chin J Integr Med Cardio/Cerebrovasc Dis. 2011; 9:917-918.

4. Hua XP, Yang Z, Li ZX, Wu RX. Effect of ShexiangTongxin dropping pill on cardiac function in patients with chronic heart failure. Chin J Integr Med Cardio/Cerebrovasc Dis. 2011; 9:143-145.

5. Chen D, Lin S, Xu W, Huang M, Chu J, Xiao F, Lin J, Peng J. Qualitative and quantitative analysis of the major constituents in Shexiang Tongxin dropping pill by HPLCQ-TOF-MS/MS and UPLC-QqQ-MS/MS. Molecules. 2015; 20:18597-18619.

6. Zhu XJ, Li HT, Yu B, Wu QB. Effects of artificial musk on myocardial ischemia in animals. Chin Pharmacol Bull. 2009; 25:951-954.

7. Wang LY, Gao X, Tong ZL, Wang XJ. Summary on pharmacological action and clinical study of the chemical composition of bear bile. Inf Tradit Chin Med. 2005; 22:30-33.

8. Tao M, Zhao WJ, Wang JW. Research progression on pharmacological action and clinical application of the chemical composition of bear bile powder. Inf Tradit Chin Med. 2013; 30:153-155.

9. Wang N, Guan P, Zhang JP, Li YQ, Chang YZ, Shi ZH, Wang FY, Chu L. Fasudil hydrochloride hydrate, a Rhokinase inhibitor, suppresses isoproterenol-induced heart failure in rats via JNK and ERK1/2 pathways. J Cell Biochem. 2011; 112:1920-1929

10. Li H, Song F, Duan LR, Sheng JJ, Xie YH, Yang Q, Chen Y, Dong QQ, Zhang BL, Wang SW. Paeonol and danshensu combination attenuates apoptosis in myocardial infarcted rats by inhibiting oxidative stress: roles of $\mathrm{Nrf} 2 / \mathrm{HO}-1$ and PI3K/Akt pathway. Sci Rep. 2016; 6:23693.

11. Ke Z, Wang G, Yang L, Qiu H, Wu H, Du M, Chen J, Song J, Jia X, Feng L. Crude terpene glycoside component from Radix paeoniae rubra protects against isoproterenol-induced myocardial ischemic injury via activation of the PI3K/ AKT/mTOR signaling pathway. J Ethnopharmacol. 2017; 206:160-169.

12. Panda S, Biswas S, Kar A. Trigonelline isolated from fenugreek seed protects against isoproterenol-induced myocardial injury through down-regulation of Hsp27 and $\alpha$ B-crystallin. Nutrition. 2013; 29:1395-403.

13. Thoonen R, Ernande L, Cheng J, Nagasaka Y, Yao V, Miranda-Bezerra A, Chen C, Chao W, Panagia M, Sosnovik DE, Puppala D, Armoundas AA, Hindle A, et al. Functional brown adipose tissue limits cardiomyocyte injury and adverse remodeling in catecholamine-induced cardiomyopathy. J Mol Cell Cardiol. 2015; 84:202-211.

14. Ryu Y, Jin L, Kee HJ, Piao ZH, Cho JY, Kim GR, Choi SY, Lin MQ, Jeong MH. Gallic acid prevents isoproterenolinduced cardiac hypertrophy and fibrosis through regulation of JNK2 signaling and Smad3 binding activity. Sci Rep. 2016; 6:34790.

15. Chen Y, Ba L, Huang W, Liu Y, Pan H, Ming YE, Shi P, Wang Y, Li S, Qi H, Sun H, Cao Y. Role of carvacrol in cardioprotection against myocardial ischemia/reperfusion injury in rats through activation of MAPK/ERK and Akt/eNOS signaling pathways. Eur J Pharmacol. 2017; 796:90-100. 
16. Thomas CJ, Lim NR, Kedikaetswe A, Yeap YY, Woodman OL, Ng DC, May CN. Evidence that the MEK/ERK but not the PI3K/Akt pathway is required for protection from myocardial ischemia-reperfusion injury by 3', 4'-dihydroxyflavonol. Eur J Pharmacol. 2015; 758:53-59.

17. Rona G. Catecholamine cardiotoxicity. J Mol Cell Cardiol. 1985; 17:291-300.

18. Patel V, Upaganlawar A, Zalawadia R, Balaraman R. Cardioprotective effect of melatonin against isoproterenol induced myocardial infarction in rats: a biochemical, electrocardiographic and histoarchitectural evaluation. Eur J Pharmacol. 2010; 644:160-168.

19. Liu JC, Baker RE, Sun C, Sundmark VC, Elsholtz HP. Activation of Go-coupled dopamine D2 receptors inhibits ERK1/ERK2 in pituitary cells. A key step in the transcriptional suppression of the prolactin gene. J Biol Chem. 2002; 277:35819-35825.

20. Uchinoumi H, Yang Y, Oda T, Li N, Alsina KM, Puglisi JL, Chen-Izu Y, Cornea RL, Wehrens XH, Bers DM. CaMKIIdependent phosphorylation of RyR2 promotes targetable pathological RyR2 conformational shift. J Mol Cell Cardiol. 2016; 98:62-72.

21. Zhong P, Quan D, Peng J, Xiong X, Liu Y, Kong B, Huang H. Role of CaMKII in free fatty acid/hyperlipidemiainduced cardiac remodeling both in vitro and in vivo. J Mol Cell Cardiol. 2017; 109:1-16.

22. Feng Y, Cheng J, Wei B, Wang Y. CaMKII inhibition reduces isoproterenol-induced ischemia and arrhythmias in hypertrophic mice. Oncotarget. 2017; 8:17504-17509. https://doi.org/10.18632/oncotarget.15099.

23. Khalilimeybodi A, Daneshmehr A, Sharif-Kashani B. Investigating $\beta$-adrenergic-induced cardiac hypertrophy through computational approach: classical and non-classical pathways. J Physiol Sci. 2017. https://doi.org/10.1007/ s12576-017-0557-5.

24. Xiong M, Jia C, Cui J, Wang P, Du X, Yang Q, Zhu Y, Wang W, Zhang T, Chen Y. Shexiang Tongxin dropping pill attenuates atherosclerotic lesions in ApoE deficient mouse model. J Ethnopharmacol. 2015; 159:84-92.

25. Yang J, Zhang XW, Deng YP, Guo M, Yao Z, Zhang ZH, Wang X. Effect of Shexiang Tongxin dripping pill on endothelial function in patients with coronary heart disease. Chin Arch Tradit Chin Med. 2016; 34:2188-2190.

26. Lin S, Chu J, Zhang L, Chen D, Xiao F, Chen H, Lin J, Chen Y, Zhu Y, Peng J. Protective effects of Shexiang Tongxin Dropping Pill on pituitrin - induced acute myocardial ischemia in rats. Mol Med Rep. 2017; 16:3125-3132.

27. Hu D, Barajas-Martínez H, Burashnikov A, Panama BK, Cordeiro JM, Antzelevitch C. Mechanisms underlying atrial-selective block of sodium channels by Wenxin Keli: experimental and theoretical analysis. Int J Cardiol. 2016; 207:326-34.

28. Qi JY, Yu J, Huang DH, Guo LH, Wang L, Huang X, Huang HD, Zhou M, Zhang MZ, Wu J. Salvianolate reduces murine myocardial ischemia and reperfusion injury via ERK1/2 signaling pathways in vivo. Chin J Integr Med. 2017; 23:40-47.

29. Ma L, Ni M, Hao P, Lu H, Yang X, Xu X, Zhang C, Huang S, Zhao Y, Liu X, Zhang Y. Tongxinluo mitigates atherogenesis by regulating angiogenic factors and inhibiting vasa vasorum neovascularization in apolipoprotein E-deficient mice. Oncotarget. 2016; 7:16194-204. https://doi.org/10.18632/oncotarget.7477. 